\title{
A Modified Multi-Pulse PPM Modulation based on PWM
}

\author{
M. Rouissat \\ Laboratory STIC of Tlemcen, Dept Electronic, Faculty of thechnology \\ Abou Bekr Belkaid University, PB 119 \\ Tlemcen, Algeria \\ ${ }^{(*)}$ E-mail:mehdi.m.rouissat@gmail.com
}

Received: 03/10/2017_Accepted: 02/04/2018

DOI: $10.7149 /$ OPA.51.2.50019

\begin{abstract}
:
In this paper, we present a new hybrid modulation, called MPDWM (Multi-Pulse Dual width Modulation). The proposed method is based on a new algorithm for symbol encoding, to enumerate the maximum number of symbols that can be generated by the combination Position-Width, which improves the data rate, and also to implement symbol synchronization, without specifying a pulse for this purpose. Principles of encoding algorithm will be clarified, the formulas of power and bandwidth requirements will be presented, comparison results to MPPM will be also analyzed.
\end{abstract}

Key words:

Optical modulation, Wireless communication, hybrid MPPM, Performance analysis

\section{REFERENCES AND LINKS / REFERENCIAS Y ENLACES}

[1] Tanveer Ahmed Bhuiyan, Samiul Hayder Choudhury, Asif Al - Rasheed, S.P. Majumder, "Performance Analysis of a Free-Space Optical Code Division Multiple Access through Atmospheric Turbulence Channel," World Academy of Science, Engineering and Technology 56 (2009).

[2] K. Kiasaleh, "Performance of APD-based, PPM free-space optical communication systems in atmospheric turbulence," IEEE Trans. Commun., 53, no. 9, pp. 1455-1461, (2005).

[3] Mohammad Noshad, Maite Brandt-Pearce, "Multilevel Pulse-Position Modulation Based on Balanced Incomplete Block Designs," Globecom 2012 - Optical Networks and Systems Symposium (2012).

[4] Abdallah Said Alahmari, "Turbo Coded Pulse Position Modulation for Optical Communications," A THESIS presented at School of Electrical and Computer Engineering Georgia Institute of Technology, (2003).

[5] Haitham S. Khallaf, Hossam M. H. Shalaby, José M. Garrido-Balsells, Seiichi Sampei, "Performance Analysis of a Hybrid QAM-MPPM Technique Over Turbulence-Free and Gamma-Gamma Free-Space Optical Channels," J. Opt. Commun. Netw. 9, 161-171 (2017).

[6] Yao Ji-Yun, "Comparison of Baseband Modulations in Visual Light Positioning," Mechanical Engineering and Technology, Advances in Intelligent and Soft Computing 125, 507-514 (2012).

[7] Roberto Ramirez-Iniguez, Sevia M. Idrus,Ziran Sun, Optical Wireless Communications: IR for Wireless Connectivity. CRC press (2008).

[8] S. Benedetto, E. Biglieri, Principles of Digital Transmission: With Wireless Applications. New York, NY, USA:Kluwer(1999).

[9] H. Selmy, H. M. H. Shalaby, Z. Kawasaki, "Bandwidth utilization enhancement for MPPM using gradual multi-pulse pulse position modulation," 2012 17th European Conference on Networks and Optical Communications, 1-6 (2012).

[10] Mehdi Rouissat, Riad A. Borsali, Mohammad E. Chikh-Bled, "A New Modified MPPM for High-Speed Wireless Optical Communication Systems," ETRI Journal 35, no. 2, 188-192 (2013). 


\section{Introduction}

Free space optical (FSO) is a powerful and potential branch of optical communication of modern age [1], it has recently attracted significant interest because of its huge unlicensed bandwidth and potential in providing high data-rates [2]. As an Intensity Modulation and Direct Detection (IM/DD) suitable modulation, PPM (Pulse Position Modulation) is considered the primary M-ary transmission technique for FSO links, since it can be implemented incoherently and does not need a threshold to make decisions at the receiver side, which is important in fading channels [3]. However, PPM presents a low spectral-efficiency, which makes dispersive channels cause interference between the time-slots, and make PPM vulnerable to inter-symbol interference (ISI). As a potential alternative, MPPM (Multi-Pulse Position Modulation) had been proposed [4]

MPPM scheme presents the best spectral efficiency in Simple Pulse Modulation category [5]; however, MPPM is not a bandwidth efficient scheme, especially for those applications of telecommunications that require an important efficiency in bandwidth use, it also suffers as PPM from synchronization problem [6,7]. On the other hand, the growing of data rate demands for wireless technologies require an alternative modulation that fulfils all these requirements and could replace the existing MPPM. Hybrid modulation techniques achieve the design goals for modern optical communication systems, namely, high power and spectral efficiencies [8]. Based on nowadays requirements, and on a hybrid solution, in the next section we introduce a new concept of modulation to improve the features of MPPM.

\section{MPDWM (Multi-Pulse Dual width Modulation)}

In the proposed approach MPDWM, the pulses take two widths (instead of one width as in the classic MPPM), so the information in a symbol is presented by the combinations Position-Width Fig. 1. The relationship between the two possible widths that a pulse can take, is a modifiable design parameter. In the presented example we took:

$$
d_{2}=(3 / 2) \cdot d_{1}
$$

$\mathrm{d}_{1}$ and $\mathrm{d}_{2}$ are the possible widths of a pulse.

In the M slots of a symbol, $w$ optical pulses will be sent, but all the pulses must take the same duration, where in the following symbol the pulses will take a different width than the previous symbol. So the width of the pulses will change alternatively. By using this feature, every symbol can be easily identified among the others. The empty $\mathrm{T}_{\mathrm{s}}$ following the last pulse will be discarded, which will make every last pulse in given symbol presents the synchronization pulse of the following symbol, in other words it will mark the beginning of a new symbol, in which pulses will take a different with, as explained before.

Each symbol starts with an empty guard slot, in order to avoid the cases where the time between adjacent pulses of two consecutive symbols is zero.

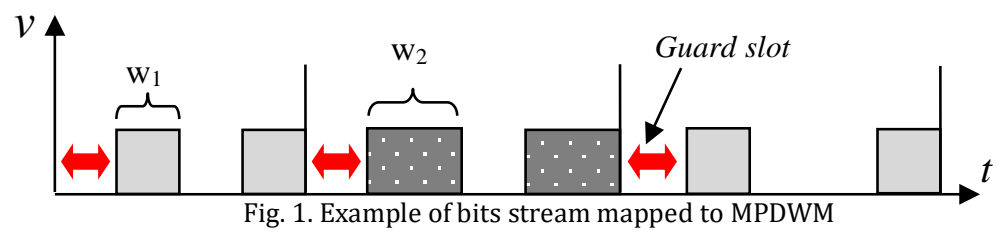

A symbol is partitioned into $M$ slots, in which (M-w) are empty and of duration $\mathrm{T}_{\mathrm{s}}$, and the other $w$ occupied slots have one of two durations; $\mathrm{T}_{\mathrm{s}}$ (one width) or $3 / 2 \mathrm{~T}_{\mathrm{s}}$ (one $\mathrm{T}_{\mathrm{s}}$ and half), based on (1). This makes the minimum number of possible occupied slots $(w+1)$, and the maximum $M$ slots, which gives an average symbol duration given by:

$$
\mathrm{T}_{\text {mean }}=\frac{\mathrm{M}+\mathrm{w}+1}{2} \mathrm{~T}_{\mathrm{s}}
$$


While in the ordinary multi-pulse pulse position modulation, the transmitted frames contain $w$ optical pulses having the same with and the same amplitude, resulting in the transmission of $\log _{2}\left(\begin{array}{l}\mathrm{M} \\ \mathrm{W}\end{array}\right)$ bits per frame [9]. By using the combination width-position; the total number of potential symbols is given by:

$$
\mathrm{L}_{\text {MPDWM }}=2\left(\begin{array}{cc}
\mathrm{M} & \mathrm{w} \\
\mathrm{w}
\end{array}\right)=2 \frac{\left(\begin{array}{ll}
\mathrm{M} & \mathrm{w}
\end{array}\right) !}{\mathrm{w} !\left(\begin{array}{ll}
\mathrm{M} & 2 \mathrm{w}
\end{array}\right) !}
$$

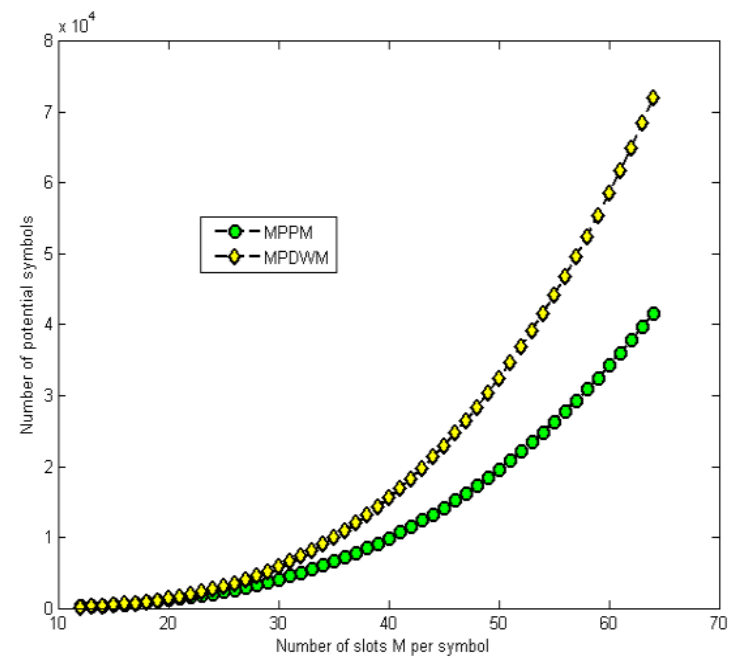

Fig. 2. Symbols that can generated, with MPPM and MPDWM with $w=3$

Fig. 2. shows the number of potential symbols that can generated by the combination position-width. we can notice that the number of possible generated symbols in MPDWM is higher compared to MPPM, especially for higher values of $M$. this advantage increase in function of the value of $M$, where for $M=67$, the possible generated symbols in MPDWM is around 1.7 times than that of MPPM.

\section{2.a. Data rate Enhancement}

The data rate achieved with the modulation MPPM is:

$$
\mathrm{R}_{\mathrm{MPPM}}=\frac{\log _{2} \mathrm{~L}_{\mathrm{MPPM}}}{\mathrm{MT}_{\mathrm{s}}}(\mathrm{bit} / \mathrm{s}) \text {; }
$$

While, the rate that we can achieved with the proposed modulation scheme is:

$$
\mathrm{R}_{\text {MPDWM }}=\frac{\log _{2} \mathrm{~L}_{\text {MPDWM }}}{\mathrm{T}_{\text {mean }}}(\mathrm{bit} / \mathrm{s})
$$

The bit rate ratio of MPDWM to MPPM is given by:

$$
\mathrm{R}_{\text {Ratio }}=\frac{\log _{2} \mathrm{~L}_{\text {MPDWM }}}{\log _{2} \mathrm{~L}_{\text {MPPM }}} \frac{2 . \mathrm{M}}{\mathrm{M}+\mathrm{w}+1}
$$

Fig.3. shows the data rate ratio of MPDWM to MPPM, function of M. The results reveal the performance of MPDWM, where the ratio for all the values of $M$ is above 1. this advantage increases as $M$ increase, where for $M=64$, the ratio is 3.3. which make the bit rate achieved by MPDWM more than three times higher than MPPM's bit rate. 


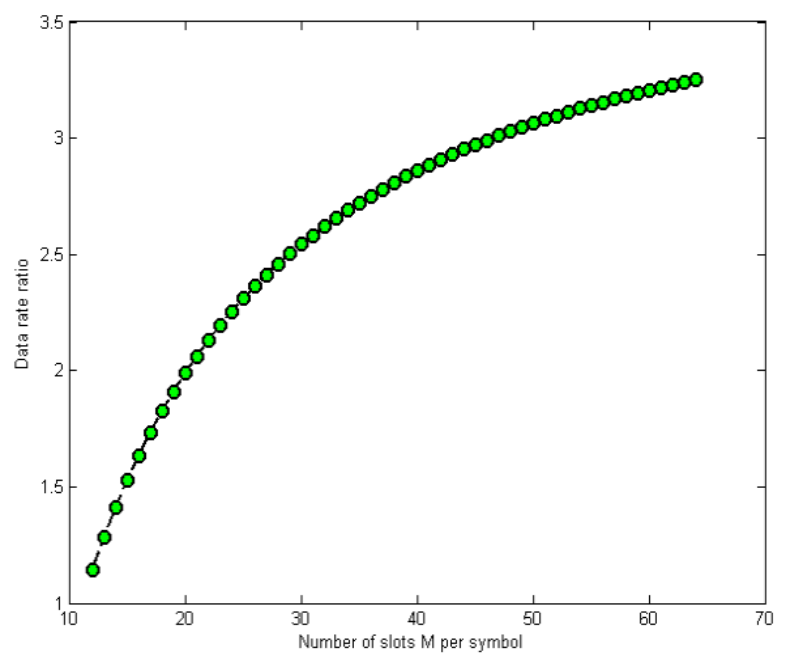

Fig. 3. Data rate ration of MPDWM over MPPM

\section{2.b. Power requirements and bandwidth efficiency}

The average power requirement of MPPM is given as:

$$
\mathrm{P}_{\text {MPPM }}=\mathrm{wd}_{1}
$$

In MPDWM, the sum of the pulses' widths can be (w.d1) or (w.d2). Based the average, the power requirement ratio of MPDWM to MPPM is given by:

$$
\mathrm{P}_{\text {ratio }}=\frac{\mathrm{w}\left(\mathrm{d}_{1}+\mathrm{d}_{2}\right)}{2} / \mathrm{w} \cdot \mathrm{d}_{1}
$$

By using (1):

$$
\begin{gathered}
\mathrm{P}_{\text {ratio }}=\frac{\mathrm{w}(5 / 2)}{2} / \mathrm{w} \\
\mathrm{P}_{\text {ratio }}=\frac{5}{4}
\end{gathered}
$$

By normalizing the average the power requirement of MPPM to that of OOK (On Off Keying)we get:

$$
\frac{\mathrm{P}_{\mathrm{MPPM}}}{\mathrm{P}_{\mathrm{OOK}}}=\frac{2 \mathrm{w}}{\sqrt{\mathrm{Md} \log _{2} \mathrm{~L}_{\mathrm{MPPM}}}}
$$

In order to find the average power requirement (NPR)of MPDWM normalized to OOK, we multiply (10) by the average N.P.R of MPPM [10]:

$$
\frac{\mathrm{P}_{\text {MPPM }}}{\mathrm{P}_{\text {OOK }}} \times \frac{\mathrm{P}_{\text {MPDWM }}}{\mathrm{P}_{\text {MPPM }}}=\frac{\mathrm{P}_{\text {MPDWM }}}{\mathrm{P}_{\text {OOK }}}
$$

Consequently, the average N.P.R for the MPDWM scheme normalized to OOK is [11]:

$$
\frac{\mathrm{P}_{\mathrm{MPDWM}}}{\mathrm{P}_{\mathrm{OOK}}}=\frac{5 \mathrm{w}}{2 \sqrt{\mathrm{Md} \log _{2} \mathrm{~L}_{\mathrm{MPPM}}}}
$$

The Band width required in MPDWM is:

$$
B_{\text {MPDWM }}=\frac{M_{\text {mean }} \cdot R_{\text {MPDWM }}}{\log _{2} L_{\text {MPDWM }}}
$$


It's important to note that the symbol duration is not a constant value for MPDWM; we must use the average symbol length (based on (2)). So the bandwidth efficiency based on this average is given by R/B:

$$
\eta_{\text {MPDWM }}=\frac{\log _{2} \mathrm{~L}_{\text {MPDWM }}}{M_{\text {mean }}}
$$

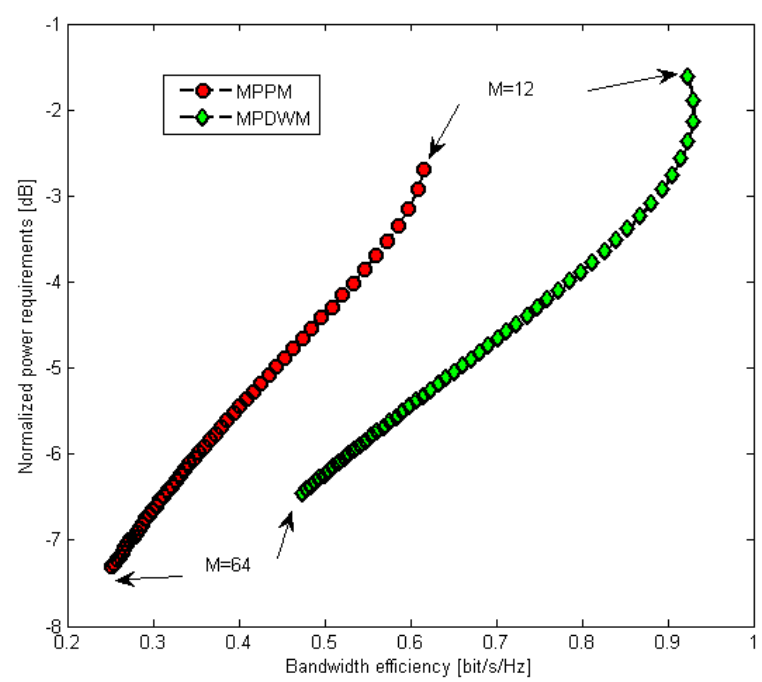

Fig.4. N.P.R based on spectral efficiency for MPPM and MPDWM, with w=3.

Fig. 4 shows the N.P.R based on the bandwidth efficiency for both MPPM and MPDWM modulation schemes. For simplicity sake, we took3 pulses per symbol, for a range values of $M$ from 12 to 64 . We can notice from the figure that the bandwidth efficiency decreases with the increase of $\mathrm{M}$, and this for both schemes, but with different speed. MPDWM presents higher efficiency compared to MPPM for all M's values. Regarding the efficiency in power use, the two schemes show an increase in this term (decrease in power requirements) as M increases, where MPPM is much efficient for all the values of $M$, with about $1 \mathrm{~dB}$ difference.

Based on the obtained results, we can say that the proposed MPDWM presents an increase of about $1 \mathrm{~dB}$ in the power requirement, which can be seen as a cost of the added improvement, in terms of that spectral efficiency, data rate and symbol synchronization.

\section{Conclusion}

Hybrid modulation techniques is a common solution for modern optical communication systems to improve the parameters that make a modulation an attractive solution. By using this philosophy, and to improve the existing MPPM, we proposed a hybrid solution based on PWM. Average power efficiency, bandwidth requirement and normalized data rate are studied after introducing the algorithm of bits encoding.

In the new concept, we allowed the pulses to take more than one width ( $\mathrm{d} 1$ and $\mathrm{d} 2$ ). Where in a given symbol, all the pulses must take the same duration, but in the following symbol the pulses will take a different width than the previous symbol.

Having always the same width, this may implement error detecting and correcting. If in a symbol the widths are set at $\mathrm{d} 2$, and one pulse is wrongly detected as $\mathrm{d} 1$, the receiver can detect this error, and can correct it.

By taking one width at a time, this feature makes every symbol easily identifiable. And it made every last pulse in given symbol presents the synchronization pulse of the following symbol.

The results also reveal that the proposed MPDWM presents an increase of about $1 \mathrm{~dB}$ in the power requirement, which can be explained as a cost of the added improvements, in terms of spectral efficiency achieved, data rate and symbol synchronization implementation. 
ÓPTICA PURA Y APLICADA

$\underline{\text { www.sedoptica.es }}$

By dint of the noteworthy improvement in three of the most important points that can make a modulation suitable for high speed communications (bandwidth, data rate and synchronization), the proposed concept may be important addition in Free Space Optical Communications field. 